\title{
Estructura y composición de la vegetación de pinares de Alturas de Pizarras en la Empresa Agroforestal Minas, Cuba
}

\section{Structure and composition of the pine forest vegetation in Alturas de Pizarra in the Minas Agroforestal Enterprise, Cuba}

\author{
Yatsunaris Alonso-Torrens*, Fernando Ramón-Hernández Martínez', Héctor Barrero-Medel', Germán López-Ibarra², \\ Nora Madanes ${ }^{3}$ y Judith Prieto-Méndez ${ }^{4}$
}

1 Universidad de Pinar del Río Hermanos Saíz Montes de Oca. Departamento Forestal, Facultad Forestal y Agronomía, Pinar del Río, Cuba. fhernandez@a upr.edu.cu, hbarrero@upr.edu.cu

* Autor de correspondencia. yatsunaris(a)
3 Universidad de Buenos Aires. Facultad de Ciencias Exactas y Naturales. Departamento de Ecología, Genética y Evolución. Laboratorio de Ecología Funcional, Laboratorio de Ecología Ambiental y Regional. Buenos Aires, Argentina. noramadanes(amail.com
4 Universidad Autónoma del Estado de Hidalgo. Instituto de Ciencias Agrícolas. Area Académica de Ciencias Agrícolas y Forestales. jud_292003@ yahoo.com.mx

\section{RESUMEN}

Esta investigación fue realizada en pinares en Alturas de Pizarra de la Empresa Agroforestal Minas, con el objetivo de determinar la estructura y composición de estos pinares, para el manejo de los mismos. Se seleccionaron dos áreas; en cada área fueron establecidas 30 parcelas circulares de 15 metros de radio; se consideraron tres estratos en la vegetación para cuantificar las especies presentes y hacer los análisis correspondientes. Se calculó la diversidad alfa y beta, se evaluó la estructura horizontal mediante el índice de importancia ecológica y la distribución por clases diamétricas, se describió la estructura vertical a través de la riqueza de especies en cada estrato, así como su posición sociológica. En ambos pinares se identificaron en total 36 familias, 47 géneros y 61 especies de plantas, 42 especies en el área de Pinus caribaea y 36 especies en el área de Pinus tropicalis, observándose diferencias en cuanto a la composición de la vegetación acompañante en sus estratos. Las especies que mayor valor de importancia ecológica alcanzaron en las áreas estudiadas fueron Pinus caribaea con 205.96 y Pinus tropicalis con 175.59, siendo la abundancia y la frecuencia relativas los parámetros que más influyeron. Las parcelas, según la composición de especies, se agruparon en cuatro grupos diferenciándose por la altitud, altura de los árboles, el área basal y la abundancia de árboles muertos presentes en las mismas. Los resultados obtenidos en este estudio, al precisar en algunos de los parámetros de esta formación vegetal en Cuba, constituyen un referente el manejo de estos pinares.

Palabras clave: diversidad alfa y beta, estrato, pino, riqueza, silvicultura.

\section{ABSTRACT}

This study was conducted in Alturas de Pizarra in the Minas Agroforestry Enterprise, in Cuba, in order to determine its composition and structure of the pine forest vegetation for future management. Two areas were selected: one corresponding to Pinus caribaea and the other to Pinus tropicalis. In each area, 30 circular plots of $15 \mathrm{~m}$ radius were established. Three strata were considered in the vegetation in order to quantify the species present and conduct the corresponding analyses. Alpha and beta diversity values were determined, the horizontal structure was evaluated by calculating the index of ecological importance and distribution by diametric classes. The vertical structure was described through the richness of species in each stratum as well as its sociological position. The two pine forests contained 36 families, 47 genera and 61 species of plants, with 42 species in Pinus caribaea area and 36 in the Pinus tropicalis area. Differences were observed in the composition of the accompanying vegetation in their strata. Pinus caribaea attained an importance value of 205.96 and Pinus tropicalis attained 175.59, relative abundance and relative frequency were the parameters that most influenced the index of ecological importance. Plots, according to the composition of species, were divided into groups of four, differing in altitude, tree height, basal area and number of dead trees found in each. By specifying some of the parameters of this vegetation type in Cuba, the results obtained in this study constitute a reference for the management of these pine groves.

KEYWORDS: alpha and beta diversity, stratum, pine, richness, silviculture. 


\section{INTRODUCCIÓN}

En Cuba, las especies del género Pinus tienen gran importancia en el plan de fomento forestal hasta el 2020; su preferencia está determinada por el rápido crecimiento que presentan y los múltiples usos que tienen sus maderas (Ministerio de Agricultura, 2013).

La existencia de pinares casi siempre está relacionada con lugares extremos donde las especies de latifolias no representan una competencia para su presencia y cuya necesidad de luz los hace excluyente de otras especies, sea por un periodo largo o solo por un tiempo limitado. Esto hace posible la prevalencia de los pinos que en general son menos exigentes con respecto a los nutrientes del suelo o algunos otros factores ecológicos (Del Risco, Toscano y Del Risco, 2000).

Según García (2006), en algunas ocasiones se han plantado especies fuera de su área de distribución natural, tal es el caso de Pinus caribaea Morelet var. caribaea Barret y Golfari, valiosa especie de rápido crecimiento, endémica del occidente del país, con gran plasticidad ecológica y considerada la especie de excelencia en los planes de reforestación a lo largo de Cuba, sobre todo en áreas naturales de Pinus tropicalis.

En las Alturas de Pizarras de la Provincia de Pinar del Río se desarrolla la formación forestal de mayor extensión: el pinar-encinar. Los primeros precursores con carácter oficial de los estudios de vegetación fueron Samek y Del Risco (1989).

Según González y Sotolongo (2007), los pinares sobre alturas de pizarra específicamente ocupan una gran parte de la provincia de Pinar del Río, crecen en pizarras (cuarcitas, areniscas y esquistos) de la formación San Cayetano, los suelos en donde se desarrollan son en general pobres, con un nivel bajo en elementos nutritivos, la profundidad de los mismos varía según la cantidad de erosión, es decir, hay suelos profundos mayormente al pie de las laderas (diluvium), por otro lado, hay lugares donde aflora la roca madre.

Según Figueroa (2002), la vegetación en alturas de pizarra está catalogada como pobre en especies, lo cual, unido a las condiciones edafoclimáticas, la ocurrencia de incendios forestales, el paso de los huracanes cada vez más frecuentes en el país, la fragmentación de los bosques y la introducción de especies exóticas, provocan variaciones en la estructura y composición de las especies que la conforman.

Varios investigadores han realizado estudios de la vegetación en las Alturas de Pizarras entre los que se pueden mencionar: León y Alain (1951, 1953 y 1957), Roig (1988), Samek y Del Risco (1989), Figueroa (2002), Benítez (2002), Valdés (2003) y González (2006).

El análisis de la estructura horizontal es insuficiente en un estudio fitosociológico, por ello, Finol (1971) propuso incluir el estudio de la estructura vertical, como una forma de describir el estado sucesional en que se encuentra cada especie.

Teniendo en cuenta que la Empresa Agroforestal Minas de Pinar del Río cuenta con un patrimonio forestal de 59 173,5 ha, del total de esta superficie cubierta de bosques los pinares en alturas de pizarras ocupan 65,5\% (Benítez y Torres, 2009). Por todo lo anteriormente mencionado para este estudio se trazaron los siguientes objetivos.

\section{OBJETIVOS}

Caracterizar la composición de la vegetación de pinares de Alturas de Pizarras en la Empresa Agroforestal Minas. Determinar la estructura horizontal y vertical de la vegetación asociada a estos pinares de alturas de pizarras en la Empresa Agroforestal Minas.

\section{MATERIALES Y MÉTODOS}

\section{Ubicación y características del área de estudio}

Se seleccionaron para este estudio dos áreas de pinares, un bosque natural de Pinus tropicalis, con una edad media de 55 años, con una pendiente promedio de $15 \%$ y una exposición SW y una plantación de Pinus caribaea de 36 años, con igual pendiente y exposición que el área anterior, según Benítez y Torres (2009). Los mismos se desarrollan en suelos ferralíticos, cuarcíticos, amarillos, con una temperatura media anual de $23,6{ }^{\circ} \mathrm{C}$ con precipitaciones promedio de $332,2 \mathrm{~mm}$ y se encuentran ubicadas en 
las siguientes coordenadas UTM: X 182 846.284-Y 299 698.8573 (Pinar de Pinus caribaea) y X 184 810.4247-Y 298423.394 (Pinar de Pinus tropicalis).

En cada área se establecieron 30 parcelas circulares de $15 \mathrm{~m}$ de radio (aproximadamente 0,07 ha), para un área total abarcada de aproximadamente 2,1 ha en cada pinar.

\section{Variables de la vegetación medidas}

Para el estudio de la vegetación se siguieron las técnicas aplicadas por James y Shugart (1970) con adecuaciones para las áreas seleccionadas. Las variables de la vegetación se tomaron en cada pinar y parcela, dividiéndose cada una en cuadrantes determinados por los puntos cardinales.

Se determinaron las especies vegetales presentes en cada área y su abundancia, la densidad de árboles/ha (Da), la densidad del sotobosque (Ds); contándose las ramas de diámetro menor o igual a $3 \mathrm{~cm}$, a la altura de $1,30 \mathrm{~m}$, en cuatro transectos desde el centro de la parcela hacia cada uno de los puntos cardinales. Posteriormente se determinó el valor por hectárea; el diámetro de los árboles a la altura de $1,30 \mathrm{~m}\left(\mathrm{D}_{1.30}\right)$; el área basal $(\mathrm{G})$ a partir de la ecuación: $G=\frac{\pi}{4} * d_{1.30}{ }^{2}$; la cobertura del dosel (Cd) y del suelo (Cs), observando a través de un tubo plástico de $4 \mathrm{~cm}$ de diámetro y $15 \mathrm{~cm}$ de largo, dividido en su extremo distal en cuatro cuadrantes. Las observaciones se realizaron en 10 puntos equidistantes, desde el centro de la parcela hacia cada uno de los puntos cardinales, cuyos valores posteriormente se promedian; la altura del dosel (H) como el promedio de las alturas $(\mathrm{m})$ de los diez árboles más altos de la parcela.

Para la nomenclatura de las especies presentes en las áreas se siguieron los criterios de Acevedo y Strong (2012).

\section{Diversidad alfa $(\alpha)$}

La diversidad (alfa) de especies forestales en cada pinar, fue estimada mediante la riqueza de especies (Magurran, 1989). Se determinaron las curvas de rarefacción para cada pinar basadas en el número de muestras mediante el software EstimateS versión 9.0.0 (Colwell, 2011). Se utili- zaron los estimadores no paramétricos basados en abundancia (CHAO 1, ACE y Cole) ya que asumen homogeneidad entre los hábitats según Magurran (2004) y la curva del número de especies representadas por un solo individuo (singletons).

\section{Diversidad beta $(\beta)$}

Para evaluar la similitud florística entre las parcelas y las poblaciones se realizó un análisis de conglomerados usando el software PC-ORD, Versión 4.17 (McCune y Mefford, 1999), mediante la medida de distancia de Sorensen cuantitativo (Bray-Curtis) y el método de asociación de los grupos fue el de Ward's, excluyéndose los pinos por ser las especies características de cada área.

\section{Estructura horizontal}

Para señalar la importancia de cada especie en el conjunto, se determinó el índice de valor de importancia ecológica (IVIE), cuyo resultado es la suma de los valores relativos de abundancia o densidad, dominancia y frecuencia de cada especie (Acosta et al., 2006; Lozada, 2010).

Los diámetros obtenidos fueron agrupados en clases diamétricas a partir de las cuales se construyó un histograma de frecuencias.

\section{Estructura vertical}

La estructura vertical se describió tomando en consideración los estratos del bosque y las especies dominantes observadas y/o registradas en cada uno de ellos. Para la estratificación vertical se consideró como estrato bajo o herbáceo a las especies que se encontraban desde el nivel del suelo hasta los $2 \mathrm{~m}$ de altura; en el estrato medio o arbustivo las que se encontraban a partir de los $2 \mathrm{~m}$ y hasta $6 \mathrm{~m}$ y en el estrato alto o arbóreo las que se encontraban a una altura superior a $\operatorname{los} 6 \mathrm{~m}$.

Para describir y analizar la distribución de las especies en sentido vertical se determinó la riqueza de especies, así como el índice de posición sociológica (PS) como expresión de la expansión vertical de las mismas (Hosokawa, 1986) para cada estrato en cada pinar. 
La PS es un índice que informa la composición florística de los distintos subestratos de la vegetación, y del papel que juegan las diferentes especies en cada uno de ellos (Hosokawa, 1986, citado por Acosta et al., 2006).

Para el cálculo del mismo se siguió la metodología de Finol (1976), se le asignó un valor fitosociológico a cada estrato, el cual se obtuvo dividiendo el número de individuos en el estrato por el número total de individuos de todas las especies $(\mathrm{VF}=\mathrm{n} / \mathrm{N})$. Siendo: $\mathrm{VF}=$ Valor Fitosociológico del subestrato; $\mathrm{n}=$ número de individuos del subestrato; $\mathrm{N}$ = número total de individuos de todas las especies.

\section{RESULTADOS}

\section{Diversidad alfa $(\alpha)$}

Se registraron 62 especies de plantas, 42 en el área de Pinus caribaea y 36 en el área de Pinus tropicalis, pertenecientes a 36 familias y 47 géneros. La familia Rubiaceae fue la de mayor riqueza de especies en ambos pinares, destacándose también las familias: Clusiaceae, Fabaceae y
Melastomataceae. Cuando se analiza la riqueza de especies mediante las curvas de rarefacción basadas en el número de muestras (Fig. 1) como el número máximo de muestras es el mismo para cada pinar, las comparaciones se realizan en la asíntota. Como los intervalos de confianza $95 \%$ para la riqueza promedio de especies se solapan entre ambas áreas, entonces no existen diferencias significativas en la riqueza de las mismas.

Para el caso de los estimadores no paramétricos (Fig. 2); todos estuvieron entre los intervalos de confianza $95 \%$ junto a los valores observados (Mao Tau) y la representatividad supera $90 \%$ (Tabla 1 ).

\section{Diversidad beta $(\beta)$}

El análisis de conglomerados en la figura 3 muestra que, aun cuando los pinares estudiados se encuentran distantes uno del otro, presentan características florísticas similares que determinan su ubicación en el dendrograma. Las parcelas según la composición de especies se agruparon fundamentalmente en cuatro grupos, dominados por: Faramea occidentalis, Sorghastrum stipoides, Lyonia myrtilloides, Cyrilla racemiflora, Byrsonima crassifolia,

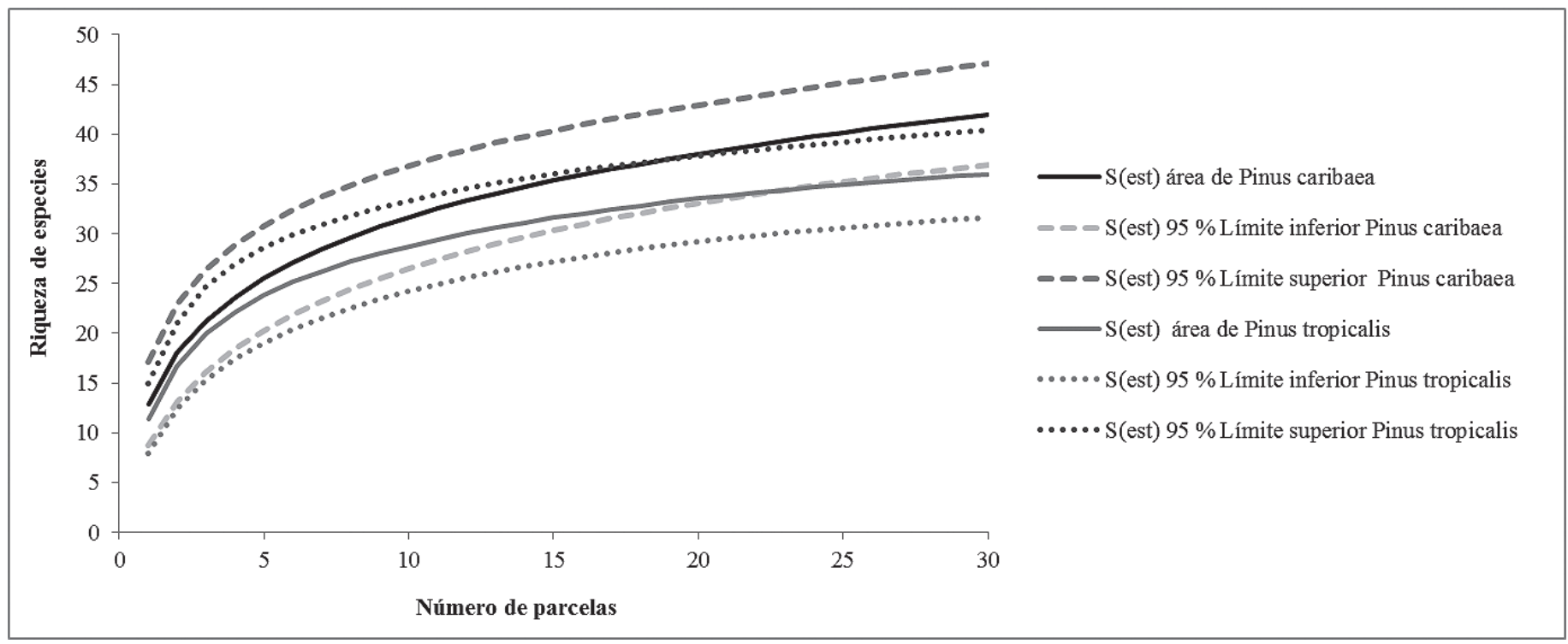

Figura 1. Curvas suavizadas de acumulación de la riqueza observada (curvas de rarefacción basadas en muestras) para cada pinar estudiado. 
TABla 1. Estimadores de riqueza y representatividad del muestreo para los pinares estudiados.

\begin{tabular}{lcccc}
\hline & \multicolumn{2}{c}{ Pinar de Pinus caribaea } & \multicolumn{2}{c}{ Pinar de Pinus tropicalis } \\
\cline { 2 - 5 } Estimadores & Riqueza & \% Representatividad & Riqueza & \% Representatividad \\
\hline S estimada & 42 & & 36 & \\
ACE & 44,46 & 94,47 & 37,83 & 95,16 \\
Chao 1 & 43,50 & 96,55 & 37,50 & 96 \\
Cole & 42,36 & 99,15 & 36,25 & 99,31 \\
\hline
\end{tabular}

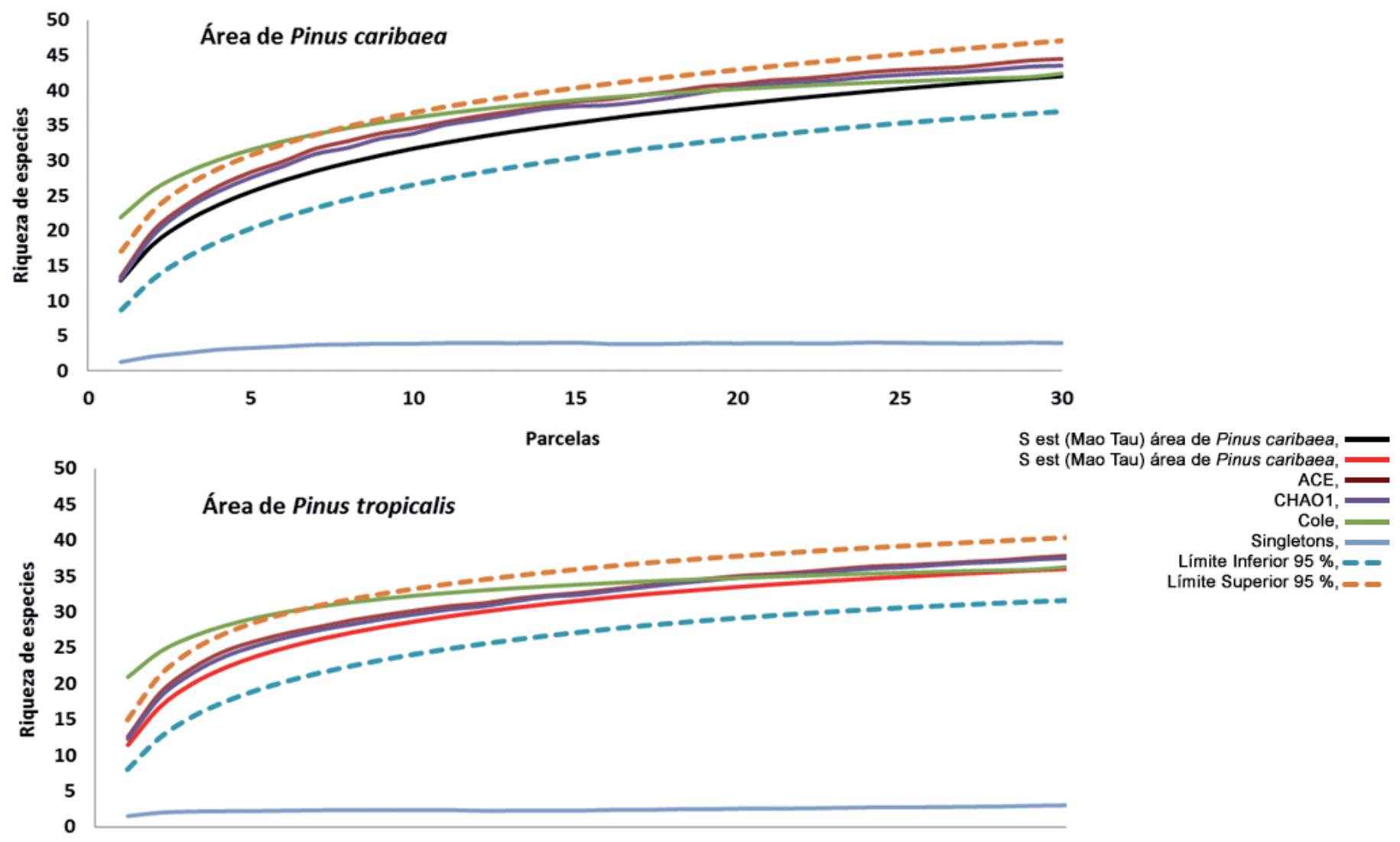

Figura 2. Curvas de acumulación de la riqueza según los estimadores no paramétricos ACE, CHAO 1 y Cole, para los pinares estudiados.

Clidemia strigillosa, Conostegia xalapensis, Davilla rugosa, Matayba apetala, Alibertia edulis, Roigella correifolia, Cyathea arborea, Brya microphylla, Parathesis cubana, Xilopia aromatica, Chrysobalanus icaco y Befaria cubensis. Se diferenciaron en cuanto a la altitud, altura de los árboles, área basal y a la cantidad de árboles muertos presentes en las mismas.

\section{Estructura horizontal de la vegetación}

El área basal del área de Pinus caribaea fue de 1,01 $\mathrm{m}^{2} / \mathrm{ha}$. En la figura 4 se muestra el histograma con la distribución de todos los árboles por cada clase diamétrica, la cual se aproxima a una distribución de frecuencia normal.

El área basal en el área de Pinus tropicalis fue de 1,39 $\mathrm{m}^{2} / \mathrm{ha}$, el tipo de distribución diamétrica se representa en 


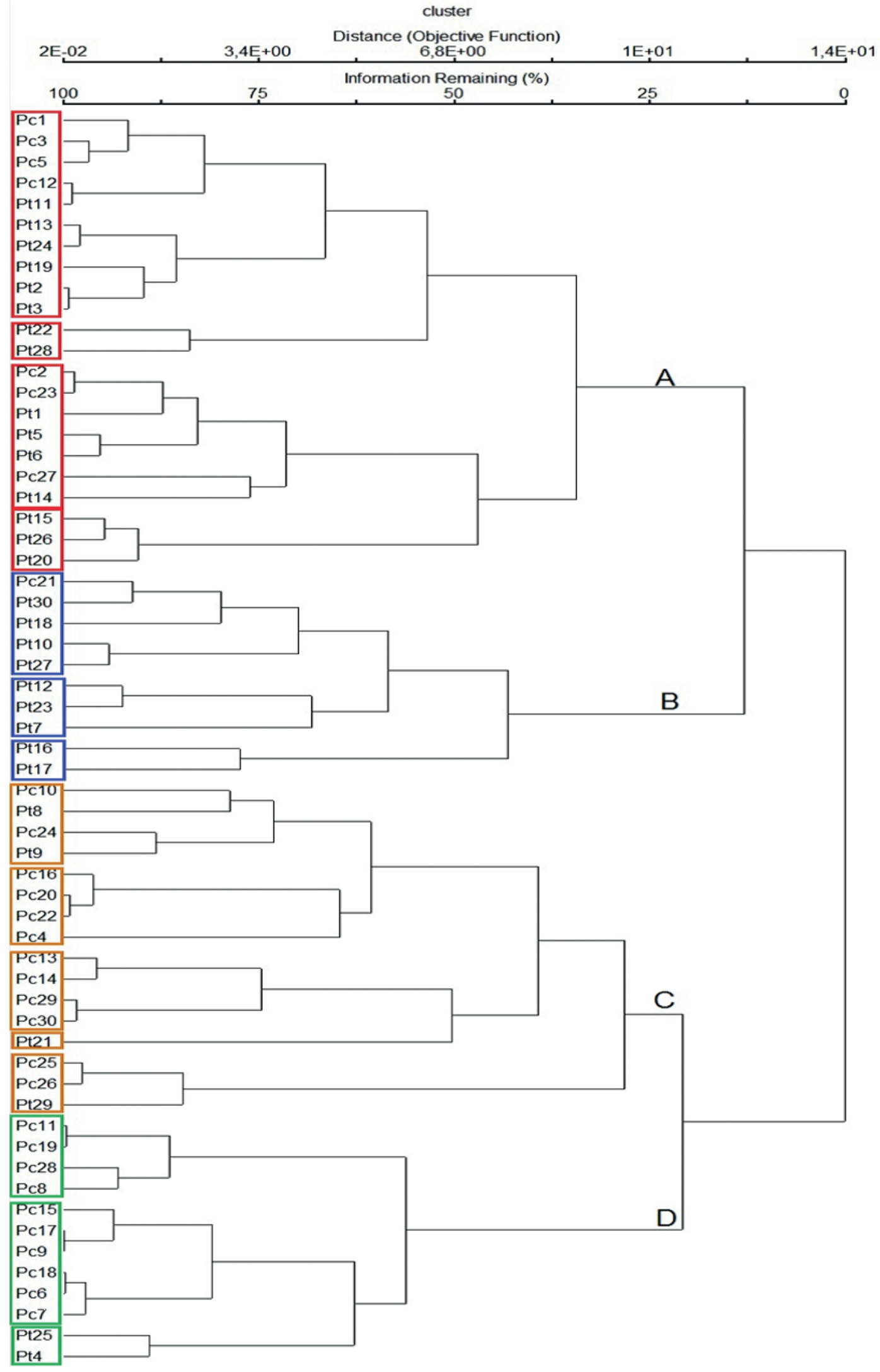

Figura 3. Dendrograma de clasificación de las parcelas para ambos pinares. 


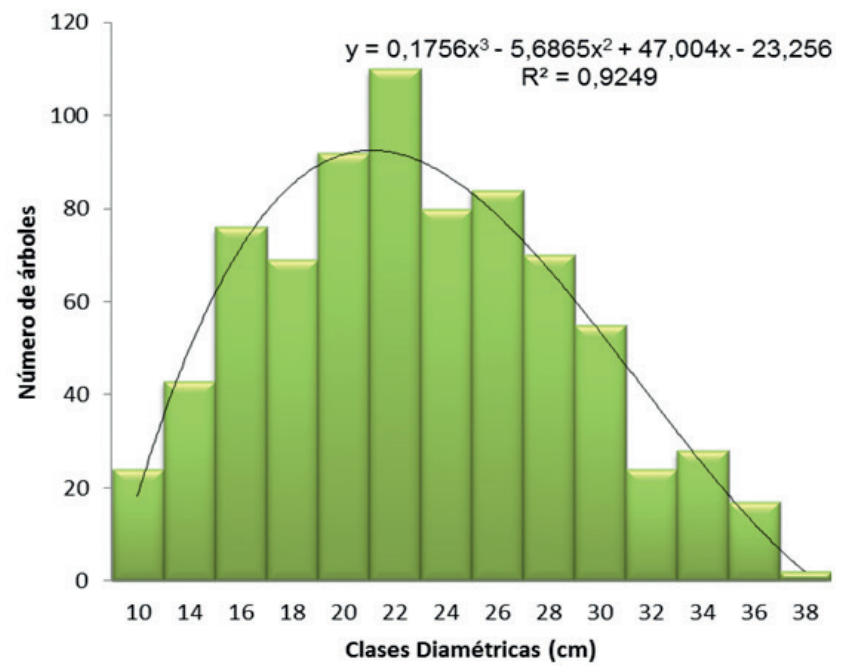

Figura 4. Distribución por clases diamétricas para el área de Pinus caribaea.

la figura 5, siendo decreciente, irregular, en forma de una J invertida, típica de un bosque natural multietáneo donde la cantidad de individuos disminuye con el diámetro creciente.

\section{Índice de valor de importancia ecológica}

Para tener una visión más amplia de la importancia de cada especie en el conjunto en ambos pinares, en la tabla 2 se ordenan de acuerdo con los valores del IVIE, las 20 primeras especies en forma decreciente y el conjunto restante lo constituye una sola categoría denominada especies raras u otras especies según Melo y Vargas (2003).

Pinus caribaea (Pincar), Pinus tropicalis (Pintro), Faramea occidentalis (Farocc), Alibertia edulis (Aliedu), Byrsonima coriacea (Byrcor), Cyrilla racemiflora (Cyrrac), Xylopia aromatica (Xylaro), Matayba apetala (Matape), Brya microphylla (Brymic), Clidemia hirta (Clihir), Roigella correifolia (Roicor),Conostegia xalapensis (Conxal), Sorghastrum stipoides (Sorsti), Cyathea arborea (Cyaarb), Jenipa americana (Jename), Parathesis cubana (Parcub), Davila rugosa (Davrug), Tabernaemontana citrifolia (Tabcit), Callophyllun pinetorum (Calpin), Abarema obovalis (Abaobo), Acoelorrhaphe wrightii (Acowri), Costaea cubensis (Coscub), Befaria cubensis (Befcub) y Curatela americana (Curame)

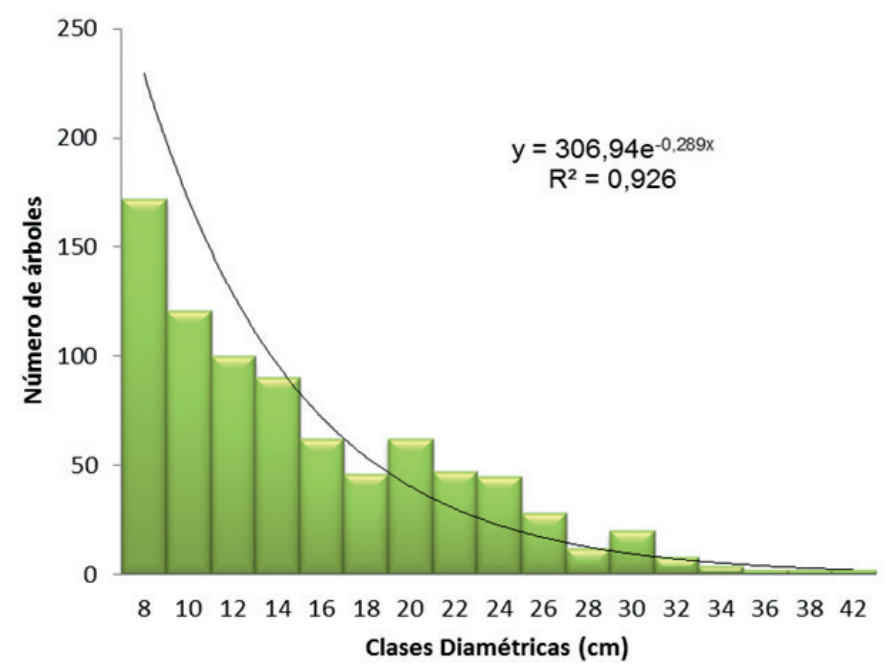

Figura 5. Distribución por clases diamétricas para el área de Pinus tropicalis.

Por otro lado, cuando se expresa por medio de un histograma la proporción del IVIE respecto a las 20 primeras especies de mayor peso ecológico, con el resto de ellas ubicada dentro de la categoría de especies raras (Fig. 6), se obtiene una primera aproximación del valor de la diversidad y de la heterogeneidad del bosque.

\section{Estructura vertical de la vegetación}

En relación con la estructura vertical de ambos pinares, se observan diferencias en cuanto a la riqueza de especies de la vegetación acompañante en sus estratos. El estrato herbáceo es el mejor representado en cuanto a la riqueza de especies, sobre todo en el área de Pinus caribaea, seguido del estrato arbustivo.

\section{Posición sociológica (PS)}

Como se puede observar en la tabla 3, el estrato herbáceo en ambos pinares es donde existe el mayor número de pies (densidad) seguido del estrato arbóreo.

\section{DISCUSIÓN}

Según los resultados obtenidos en este estudio, el número total de especies inventariadas representa $11,33 \%$ de lo planteado por Samek (1973) para las Alturas de Pizarras; 
TABLA 2. Índice de valor de importancia ecológica de las especies en los pinares estudiados.

\begin{tabular}{|c|c|c|c|c|c|c|c|c|c|}
\hline \multicolumn{5}{|c|}{ Pinar de Pinus caribaea } & \multicolumn{5}{|c|}{ Pinar de Pinus tropicalis } \\
\hline Especie & Ar & $\mathrm{Dr}$ & $\mathrm{Fr}$ & IVIE & Especie & Ar & $\mathrm{Dr}$ & $\mathrm{Fr}$ & IVIE \\
\hline Pincar & 105,3 & 0,30 & 77,3 & 182,87 & Pintro & 135,5 & 0,40 & 103,9 & 239,79 \\
\hline Farocc & 54,4 & 0,02 & 17,9 & 72,35 & Farocc & 22,3 & 0,02 & 22,4 & 44,81 \\
\hline Aliedu & 17,3 & 0,00 & 17,5 & 34,80 & Vaccub & 23,2 & 0,00 & 14,0 & 37,14 \\
\hline Byrcor & 11,5 & 0,00 & 12,4 & 23,84 & Cyaarb & 14,5 & 0,00 & 10,2 & 24,71 \\
\hline Cyrrac & 2,3 & 0,00 & 20,6 & 22,87 & Parcub & 7,6 & 0,00 & 14,5 & 22,07 \\
\hline Xylaro & 7,4 & 0,08 & 15,1 & 22,59 & Matape & 7,5 & 0,00 & 13,7 & 21,21 \\
\hline Matape & 13,8 & 0,04 & 8,6 & 22,49 & Byrcor & 6,4 & 0,00 & 14,8 & 21,18 \\
\hline Brymic & 13,3 & 0,00 & 5,9 & 19,25 & Cyrrac & 9,4 & 0,00 & 8,4 & 17,72 \\
\hline Clihir & 9,3 & 0,00 & 9,9 & 19,16 & Conxal & 11,1 & 0,00 & 5,2 & 16,32 \\
\hline Roicor & 8,3 & 0,00 & 8,1 & 16,36 & Sorsti & 10,4 & 0,00 & 5,2 & 15,64 \\
\hline Conxal & 8,0 & 0,00 & 7,0 & 15,04 & Roicor & 8,9 & 0,00 & 5,2 & 14,07 \\
\hline Sorsti & 8,7 & 0,00 & 6,2 & 14,89 & Davrug & 5,8 & 0,00 & 7,8 & 13,60 \\
\hline Cyaarb & 9,1 & 0,00 & 4,8 & 13,89 & Xylaro & 2,3 & 0,00 & 8,4 & 10,62 \\
\hline Jename & 1,5 & 0,06 & 11,8 & 13,44 & Clihir & 5,9 & 0,00 & 4,5 & 10,34 \\
\hline Parcub & 4,4 & 0,00 & 8,8 & 13,19 & Aliedu & 3,6 & 0,00 & 6,1 & 9,71 \\
\hline Davrug & 5,0 & 0,00 & 7,3 & 12,37 & Brymic & 5,5 & 0,00 & 4,2 & 9,68 \\
\hline Tabcit & 3,0 & 0,00 & 5,7 & 8,68 & Geoine & 1,4 & 0,00 & 6,6 & 7,95 \\
\hline Calpin & 1,6 & 0,32 & 5,1 & 7,00 & Coscub & 4,6 & 0,00 & 3,1 & 7,64 \\
\hline Abaobo & 0,6 & 0,05 & 6,1 & 6,77 & Befcub & 2,8 & 0,00 & 4,5 & 7,30 \\
\hline Acowri & 3,3 & 0,00 & 3,1 & 6,33 & Curame & 1,4 & 0,00 & 5,7 & 7,11 \\
\hline
\end{tabular}

$\mathrm{Ar}=$ Abundancia relativa, $\mathrm{Dr}$ = densidad relativa, $\mathrm{Fr}=$ Frecuencia relativa; IVIE = Índice de Valor de Importancia Ecológica

en el área de Pinus caribaea es superior al obtenido por Benítez (2002) en plantaciones de dicha especie y en áreas de la Empresa Forestal Integral (EFI) Minas (26 especies), en las cuales se efectuaron diferentes tratamientos de preparación del terreno para favorecer la regeneración natural; mientras que el área de estudio es una plantación en estado de latizal alto en la que hace varios años no se efectúa ningún tratamiento, razones que pudieron haber incidido en esa diferencia.

Por otra parte, el número total de especies inventariadas en el área de Pinus tropicalis se asemeja al obtenido por Figueroa (2002), quien obtuvo un valor medio de 15 especies y un máximo de 33 para esta formación en condiciones naturales en las alturas de pizarras en áreas de la EFI La Palma y en áreas de la localidad conocida por Bajas de la EFI Minas.

El muestreo fue representativo ya que los valores del conjunto de estimadores se comportan de forma similar y presentan valores cercanos a los observados. Además, la curva de los singletons es asintótica, por lo que se considera que se ha obtenido un buen muestreo según Villarreal et al. (2006). Por esta razón, el incremento en el esfuerzo de muestreo no causa aumentos sustanciales en la riqueza de especies. El estimador más acertado para 
TABLA 3. Valor fitosociológico de cada estrato en ambos pinares.

\begin{tabular}{ccccc}
\hline Pinar & Estrato & N/ha & VF (\%) & VF simplificado \\
\hline Pc & Herbáceo & 1341 & 66,45 & 6,65 \\
Pc & Arbustivo & 314 & 15,57 & 1,56 \\
Pc & Arbóreo & 363 & 17,98 & 1,8 \\
Pt & Herbáceo & 1272 & 67,1 & 6,71 \\
Pt & Arbustivo & 229 & 12,08 & 1,21 \\
Pt & Arbóreo & 395 & 20,82 & 2,08 \\
\hline
\end{tabular}

N/ha = Número de pies por hectárea, VF = Valor fitosociológico.

cada pinar fue CHAO 1, el cual predice valores muy similares a los encontrados y el porcentaje de representatividad supera $96 \%$.

Las diferencias entre parcelas en cuanto a la composición de las especies presentes en las mismas pudieran estar dadas por la altitud a las que se encuentran, variando desde los $46 \mathrm{~m}$ snm en el área de Pinus caribaea hasta los 133 m snm en el área de Pinus tropicalis, ya que según Körner (2000), las dimensiones verticales de las montañas producen gradientes climáticos con variaciones abruptas o graduales en temperatura, humedad relativa, radiación solar y precipitación, lo cual provoca un efecto en la distribución y abundancia de la flora. Esto coincide con lo planteado por Bermeo (2010), quien argumenta que la distribución y composición de especies varía en las zonas montañosas en función de la altitud y la cercanía a las partes de agua donde pueden encontrarse otras especies acidófilas, según Álvarez y Varona (2006), favoreciendo o disminuyendo la aparición de especies.

El estrato herbáceo es el más rico sobre todo en el área de Pinus caribaea, coincidiendo con Valdés (2003), quien plantea que cuando se tratara de plantaciones de esta espe-

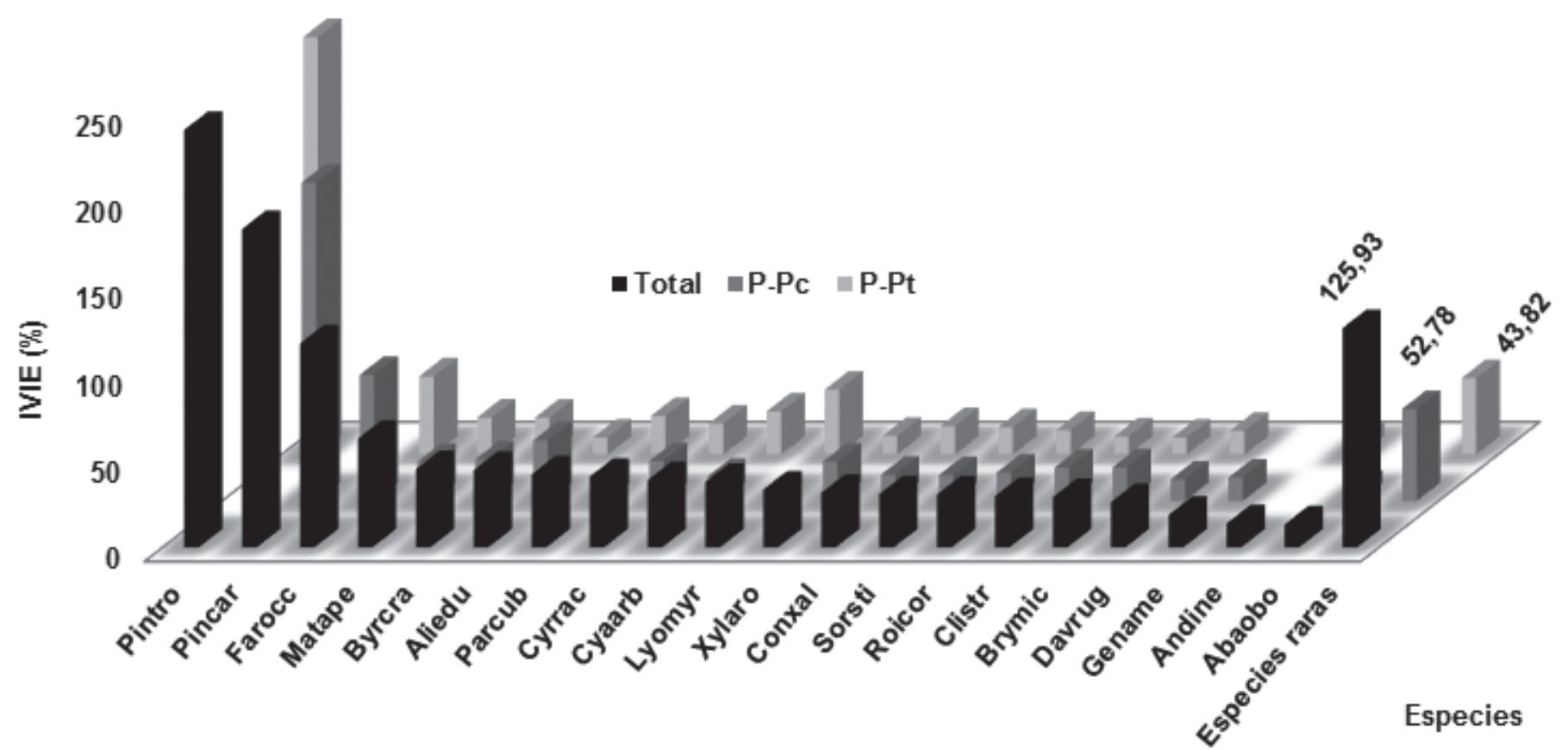

Figura 6. Comportamiento del índice de valor de importancia ecológica (IVIE) en los pinares estudiados, para las 20 primeras especies y las especies raras.

Área de Pinus caribaea (P-Pc), área de Pinus tropicalis (P-PT). Pinus tropicalis (Pintro), Pinus caribaea (Pincar), Faramea occidentalis (Farocc), Matayba apetala (Matape), Byrsonima crassifolia (Byrcra), Alibertia edulis (Aliedu), Parathesis cubana (Parcub), Cyrilla racemiflora (Cyrrac), Cyathea arborea (Cyaarb), Lyonia myrtilloides (Lyomyr), Xylopia aromatica (Xylaro), Conostegia xalapensis (Conxal), Sorghastrum stipoides (Sorsti), Roigella correifolia (Roicor), Clidemia strigillosa (Clistr), Brya microphylla (Brymic), Davilla rugosa (Davrug), Genipa americana (Gename), Andira inermis (Andine) y Abarema obovalis (Abaobo). 
cie y la misma fuera muy joven, este estrato se encontraría desarrollado, comenzando a desaparecer en la medida en que el pino crece. Cuando comienzan a realizarse las actividades silviculturales, como los raleos y las cortas selectivas, este estrato vuelve a estabilizarse y a pesar de las afectaciones, estaría más desarrollado y sería más homogéneo que cuando se presentara en pinares naturales.

En el área de Pinus tropicalis el estrato herbáceo es también el más rico, en esta área existen varias zonas donde la cobertura del dosel es baja y favorecería la entrada de luz a los estratos inferiores del bosque, la formación de este estrato y del arbustivo, a pesar de que se pueden encontrar algunas zonas donde la densidad de copa es alta y este estrato está empobrecido, reduciéndose el número de especies y de individuos.

En estos pinares el estrato arbustivo está bien desarrollado sobre todo en el área de Pinus caribaea, aunque no se encuentra de forma homogénea, desarrollándose mejor en zonas cercanas a las galerías o arroyos. Estos resultados coinciden con lo planteado por Berazaín, Areces, Lazcano y González (2005) en que en los bosques de pino los estratos arbustivo y herbáceo están bien desarrollados con pocas epífitas y trepadoras, a pesar de que en ambos pinares abunda Davilla rugosa.

De los tres estratos analizados el que más incidencia ha tenido en la posición sociológica es el herbáceo seguido del arbustivo, debido a que se han detectado mayor cantidad de individuos en los mismos, sobre todo por la regeneración natural de algunas de las especies.

Entre las especies que se encuentran en los 3 estratos están: Pinus caribaea, Calophyllum pinetorum, Genipa americana, Abarema obovalis, Faramea occidentalis, Matayba apetala y Xilopia aromatica; garantizando así según Acosta et al. (2006) su lugar en la estructura y composición del bosque. Las últimas tres poseen una posición sociológica regular, ya que presentan en el estrato inferior un número de individuos mayor o igual al de los estratos subsiguientes, según lo planteado por Acosta et al. (2006) y, de estas, Faramea occidentalis es la que alcanzó el máximo valor en cuanto a la posición sociológica, destacándose su presencia en esta área.
Se observó en estos pinares que la regeneración natural, aunque no fue una de las variables de la vegetación medidas, también manifiesta variaciones locales; lo cual pudiera estar dado por la acumulación de acículas en el suelo, siendo abundante en algunos lugares; la cobertura del suelo por las especies herbáceas y la presencia o no de cerdos en la zona.

Como se puede apreciar, el mayor peso ecológico lo tienen las especies dominantes y predominantes en este tipo de formación, por lo que se está caracterizando un ecosistema boscoso con tendencia a la homogeneidad según Kageyama (1994).

\section{CONCLUSIONES}

Este estudio permitió conocer la composición de especies de pinares sobre Alturas de Pizarra donde los pinos son las especies dominantes, pero debido a los manejos silvícolas a los que han estado sometidos, conviven con varias especies en todos los estratos, llegando a tal magnitud en algunas áreas que pudiera dar idea de una sucesión, con la disminución de ambas especies de pinos y observándose poca regeneración natural de las mismas. En los pinares estudiados no existieron diferencias significativas en las especies vegetales asociadas según la tendencia de las curvas de rarefacción y los estimadores no paramétricos, prediciendo los mismos valores muy similares a los observados con más de $99 \%$ de representatividad. En ambas áreas los dos pinos mostraron el mayor índice de valor de importancia ecológica (IVIE); el estrato herbáceo fue el mejor representado seguido del estrato arbustivo en cuanto a la riqueza de especies, siendo los de mayor incidencia en la posición sociológica. El estrato herbáceo en ambos pinares es donde existió el mayor número de pies seguido del arbóreo.

\section{RECONOCIMIENTOS}

Expresamos nuestros agradecimientos a los siguientes especialistas por su apoyo en el desarrollo de este proyecto: a Orlando y Juan del cuerpo de guardabosques del Tibis; $\mathrm{H}$. Benítez y JM. Torres de la EFI Minas, Ibrahín del cuerpo de guardabosques del municipio Minas de Matahambre; A. Plasencia, Elvis y Geldys del Museo de Ciencias Naturales 
de Pinar del Río, R. Sotolongo de la Universidad de Pinar del Río; JL. Rodríguez de la Universidad de Granma y G. Golstein de la Universidad de Buenos Aires.

\section{REFERENCIAS}

Acevedo, P. y Strong, M. (2012). Catalogue of seed plants of the West Indies. Smithsonian Institution Scholarly Press.

Acosta, V., Araujo, P e Iturre, M. (2006). Caracteres estructurales de las masas. Serie Didáctica No 22. Universidad Nacional de Santiago del Estero. 35 p. Recuperado de http://www.virtual.unal.edu.co/cursos/sedes/manizales / 4010014 / Contenidos / Capitulo6/ Pages/6.2/62Definicion_estructura_organizacional.htm

Álvarez, P. A. y Varona, J. C. (2006). Silvicultura. La Habana: Ciencia y Técnica.

Benítez, H. y Torres, J. M. (2009). Proyecto de ordenación de la Empresa Forestal Integral Minas. 82 p.

Benítez, H. (2002). Regeneración natural de Pinus caribaea var. caribaea mediante talas rasas en fajas alternas. Disertación doctoral. Programa doctoral conjunto "Desarrollo Sostenible de Bosques Tropicales".

Berazaín, R., Areces, F., Lazcano, J. C. y González, L. R. (2005). Lista roja de la flora vascular cubana. Documentos del Jardín Botánico Atlántico (Gijón), 4, 1- 86.

Bermeo, D. F. (2010). Determinación y caracterización de tipos funcionales de plantas (TFPs) en bosques secundarios dentro de un gradiente altitudinal y su relación con variables bioclimáticas. Tesis de maestría no publicada. Catie. Costa Rica.

Colwell, R. K. (2011). Estimates: statistical estimation of species richness and shared species from samples (Version 9). [Software de cómputo]. http://purl.oclc.org/estimates.

Del Risco, E.; Toscano, B y Del Risco, E. (2000). Los Pinos cubanos y su diversidad florística. Revista Flora y Fauna, 1, 23-26.

Figueroa, C. (2002). Ecología y conservación de Pinus tropicalis en bosques naturales de las alturas de pizarras. Disertación doctoral. Programa doctoral conjunto "Desarrollo Sostenible de Bosques Tropicales”.

Finol, H. (1971). Nuevos parámetros a considerarse en el análisis estructural de las selvas vírgenes tropicales. Revista Forestal Venezolana, 14 (21), 29-42.
Finol, H. (1976). Métodos de regeneración natural en algunos tipos de bosques venezolanos. Revista Forestal Venezolana, 19 (26), 17-44.

García, Y. (2006). Estrategia de conservación intraespecífica para Pinus caribaea Morelet var. caribaea Barret y Golfari. Disertación doctoral. Programa doctoral conjunto "Desarrollo Sostenible de Bosques Tropicales".

González, E y Sotolongo, R. (2007). Ecología forestal. La Habana: Editorial Félix Varela.

González, M. (2006). Influencia de la densidad de plantación en la economía y la ecología de Pinus caribaea Morelet var. caribaea en las Alturas de Pizarras de Pinar del Río, Cuba. Disertación doctoral. Programa doctoral conjunto “Desarrollo Sostenible de Bosques Tropicales".

Hosokawa, R. T. (1986). Manejo e economia de florestas. Roma: FAO.

James, F. C. y Shugart, H. (1970). A quantitative method of habitat description. Audubon Field Notes, 24, 727-736 p.

Kageyama, P. (1994). Revegetacao de areas degradadas: Modelos de consociacao con alta diversidade. En II Simposio internacional sobre recuperacao de áreas de gradadas. Foz de Iguacu. $559-576$ p.

Körner, C. (2000). Why are there global gradients in species richness? Mountains might hold the answer. Trends in Ecology and Evolution, 15, 513-514.

León, H. y Alain, H. (1951). Flora de Cuba. Contribuciones Ocasionales del Museo de Historia Natural del Colegio “De La Salle”, 2, 1- 456.

León, H. y Alain, H. (1953). Flora de Cuba. Contribuciones Ocasionales del Museo de Historia Natural del Colegio “De La Salle”, 3, 1- 502.

León, H. y Alain, H. (1957). Flora de Cuba. Contribuciones Ocasionales del Museo de Historia Natural del Colegio "De La Salle", 4, 1-556.

Lozada, J. (2010). Consideraciones metodológicas sobre los estudios de comunidades forestales. Revista Forestal Venezolana, 54 (1), 77-88.

Magurran, A. (1989). Diversidad ecológica y su medición. España: Vedrá.

Magurran, A. E. (2004). Comparative studies of diversity. En A. E. Magurran. Ecological diversity and its measurement 
(Cap. 5 144-161). Malden, MA, EUA; Oxford, R.U.; Victoria, Australia: Blackwell Science Ltd.

McCune, B. y Mefford, M. J. (1999). Multivariate analysis of ecological data. PC-Ord for Windows (Version 4.0). [Software de cómputo]. Glenenden Beach, Oregon, EEUU: MjM Software.

Melo, O y Vargas, R. (2003). Evaluación ecológica y silvicultural de ecosistemas boscosos. Colombia: Universidad del Tolima.

Ministerio de Agricultura. (2013). Plan de fomento forestal para la etapa 2013-2020.

Roig, J. T. (1988). Diccionario botánico de nombres vulgares cubanos. La Habana: Editorial Ciencia y Técnica.

Samek, V. (1973). Regiones fitogeográficas de Cuba. Academia de Ciencias de Cuba. Serie Forestal, 15, 1-60

Samek, V y Del Risco, E. (1989). Los Pinares de la Provincia de Pinar del Río, Cuba. Estudio sinecológico. Cuba: Editorial Academia.
Valdés, N. (2003). Efecto de la tala rasa sobre la vegetación leñosa en los ecosistemas de pinares en la unidad silvícola San Andrés perteneciente a la EFI La Palma. Disertación doctoral. Programa doctoral conjunto "Desarrollo Sostenible de Bosques Tropicales".

Villarreal, H., Álvarez, M., Córdoba, S., Fagua, F. G., Gast, F., Mendoza, H., Ospina, M. y Umaña, A. M. (2006). Manual de métodos para el desarrollo de inventarios de biodiversidad ( $2^{\mathrm{a}}$ ed.). Programa de Inventarios de Biodiversidad. Bogotá, Colombia: Instituto de Investigación de Recursos Biológicos Alexander von Humboldt.

Manuscrito recibido el 11 de enero 2016

Aceptado el 16 de agosto de 2016.

Este documento se debe citar como:

Alonso-Torrens, Y., Hernández-Martínez, F. R., Barrero-Medel, H., López-Ibarra, G., Madanes, N. y Prieto-Méndez, J. (2016). Estructura y composición de la vegetación de pinares de Alturas de Pizarras en la Empresa Agroforestal minas, Cuba. Madera y Bosques, 22 (3), 75-86. 